\title{
Neutral Injection Heating in Fusion Devices
}

\author{
Beam Production and Heating Mechanism
}

\author{
P. Lomas, Abingdon
}

(JET Joint Untertaking)

The plasma in most magnetic fusion configurations is either insufficiently hot or cannot be sustained for sufficiently long periods. In others we want to produce a particular distribution in real or velocity space. The injection of energetic particles can contribute to solving both kinds of problem, providing a source of heat and matter. In magnetic fusion plasmas it is necessary for the energetic particles to cross magnetic fields and yet be stopped in the plasma; this is conveniently done with a beam of neutral atoms.

\section{Overview}

In the last decade neutral beam heating has progressed from pilot experiments employing beams of only a few tens of $\mathrm{kW}$ to being a tried and trusted technique employing beams of many $\mathrm{MW}$. The technique has found many applications in fusion research and contributed significantly to the understanding of the physics of confined plasma. Perhaps the most notable achievement to date is the temperature of $7 \mathrm{keV}$ (70 MK) reached in the tokamak PLT at Princeton. Shortly, powerful neutral beam heating will be applied to JET ${ }^{1}$ ), in addition to powerful radiofrequency heating $\left.{ }^{2}\right)$, with the aim of producing even higher temperatures.

A long article could be written on the experiments that have been made possible with neutral beams, but this article will concentrate on how neutral beams are produced and how they interact with plasma.

The elements of a neutral beam heating system are shown in Fig. 1. lons produced in a plasma source are accelerated by a set of high voltage grids and neutralised. Unwanted ions are dumped, while the beam of neutral atoms passes through the magnetic fields of the fusion device and enters the plasma. Here the beam is ionised by the plasma and the resulting energetic ions are confined by the magnetic fields. These energetic ions slow down and give up their energy to the plasma. As the next two sections will show, the processes involve atomic physics, electronics, orbit theory and collision theory. These are all well understood.

\section{Generation of lons}

The first stage in the production of a neutral beam is the generation of some tens of amps of hydrogen ions with which to illuminate the high voltage acceleration grids. This is done in the plasma source illustrated in Fig. 2. This is simply a chamber containing a thermionically emitting cathode, an anode and an inlet for gas. The gas is ionised by the thermionic electrons and a plasma is formed with an electrostatic plasma sheath surrounding the cathode. Most of the discharge voltage appears across this sheath and a few $100 \mathrm{~A}$ of electrons are drawn out of the cathode and accelerated to energies of about $50 \mathrm{eV}$. These primary electrons ionise and dissociate the incoming gas molecules, producing neutral atoms, molecular ions and protons by the reactions listed in the table.

Reactions in the Plasma Source Ionisation of molecule $\mathrm{H}_{2}+\mathrm{e}=\mathrm{H}_{2}^{+}+2 \mathrm{e}$ Dissociation of molecule $\mathrm{H}_{2}+\mathrm{e}=2 \mathrm{H}^{\circ}+e$ Ionisation of atom $\quad \mathrm{H}+\mathrm{e}=\mathrm{H}^{+}+2 \mathrm{e}$ Dissociation of ionic molecule $\quad \mathrm{H}_{2}^{+}+\mathrm{e}=\mathrm{H}^{0}+\mathrm{H}^{+}+\mathrm{e}$ Formation of $\mathrm{H}_{3}^{+} \quad \mathrm{H}_{2}^{+}+\mathrm{H}_{2}=\mathrm{H}_{3}^{+}+\mathrm{H}$ Dissociation of $\mathrm{H}_{3}^{+} \quad \mathrm{H}_{3}^{+}+\mathrm{e}=\mathrm{H}_{2}^{+}+\mathrm{H}+\mathrm{e}$ Negative ion formation $\mathrm{H}_{2}^{*}+\mathrm{e}=\mathrm{H}^{-}+\mathrm{H}$ (* indicates vibrationally excited molecule)

In order to increase the utilisation of the primary electrons, the walls of the chamber are often shielded either electrostatically or magnetically. Magnetic designs also prevent the loss of ionic products except to the acceleration grids where they are needed. With good design, a few amps. of ions can be pro-

Fig. 1 - The elements of a neutral beam heating system.

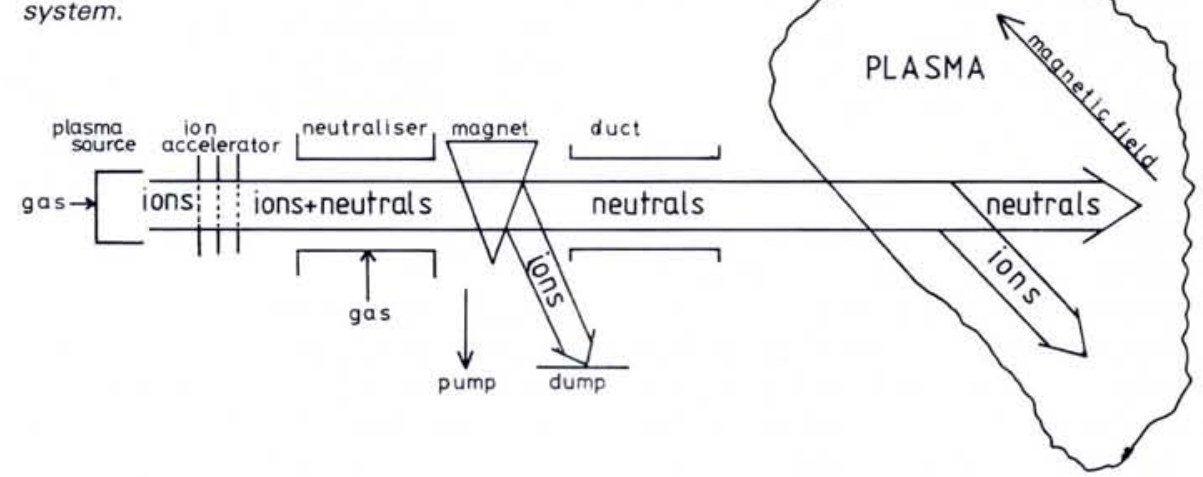

duced with the expenditure of only $1 \mathrm{~kW}$ of input power.

\section{Acceleration of lons}

The low energy ions produced by the plasma source are accelerated by high voltage grids to energies in the range $10-100 \mathrm{keV}$ as required. These grids consist of an array of aligned apertures in three or more electrodes. The simplest configuration is a single aperture in three electrodes as shown in Fig. 3. Ions from the plasma source are accelerated from the positive electrode through the earth electrode, whereas electrons are repelled back into the plasma source. Thus a meniscus or sheath is formed separating the essentially electric field-free source plasma, from the electric fields between the electrodes. Downstream of the earth electrode, the ion beam ionises the background gas to form another plasma. This neutralises the space charge of the beam, but in order to prevent the electrons in this plasma from being accelerated backwards into the plasma source, it is necessary to reverse the electric field near the electrode by means of a small negative potential on the second electrode.

The acceleration of ions in the space charge fields of this configuration is identical to the acceleration of electrons in a vacuum tube diode, except that the emitting boundary is not fixed. It can bulge out from the electrode as illustrated in the figure, with a curvature determined by the ratio $I V^{3 / 2}$, which is known as perveance. Here $l$ is the ion beam current and $V$ is the applied voltage. Since the source plasma is highly 


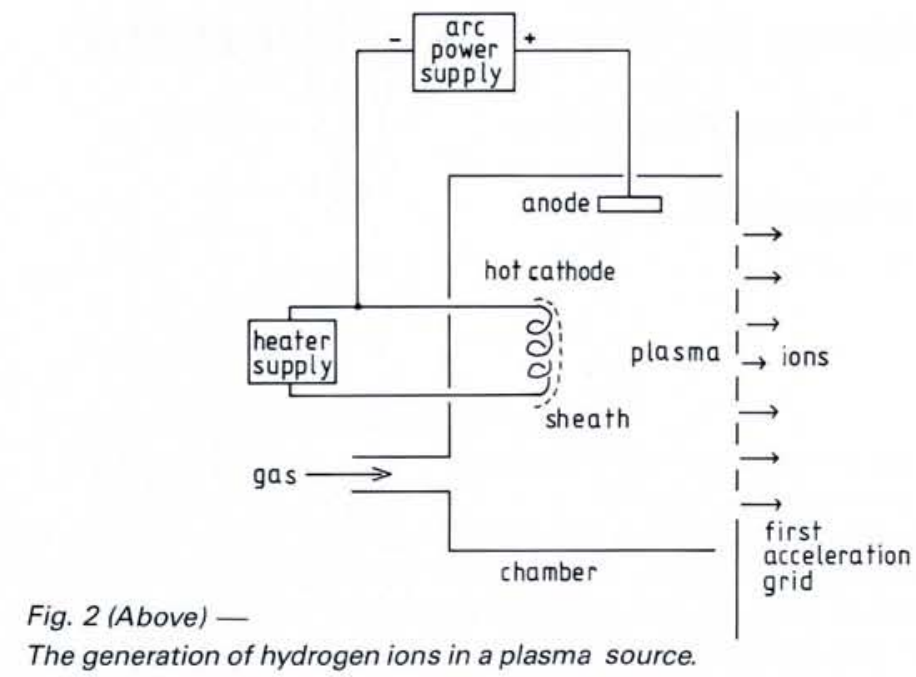

Fig. 3 (Right) - A single aperture, three electrode ion acceleration system.

conducting, the electric field in the vacuum side of the sheath is everywhere perpendicular to the sheath. Thus the ion beam is initially strongly convergent, for the curvature illustrated, i.e. there is a lens effect.

The change in electric field between the accelerating gap and the region downstream gives rise to an electrostatic lens effect, because equipotentials tend to bulge out of the aperture. This lens is divergent, and fixed by electrode geometry.

These two lenses can be made to cancel by choosing the appropriate optimum perveance which is inversely related to the acceleration length, the limit being set by metal to metal breakdown. The maximum ion current in the beam is then limited to typically $300 \mathrm{~mA}$ at 50 $\mathrm{keV}$. In order to accelerate a useful current it is necessary to incorporate many such apertures in the grid. A typical ion source may have electrodes of a few $100 \mathrm{~cm}^{2}$ area, with as many as 1000 apertures and be able to deliver $50 \mathrm{~A}$ of protons with energies of $50 \mathrm{keV}$.

The divergence of the ion beam from each aperture can be made very low by careful design of the electrostatic optics, and is typically in the range 0.5 $1.5^{\circ}$. The beamlets from different parts of the grid are steered to meet at a focus some metres away. In order to achieve this the grids have to be constructed very accurately, and this accuracy must be maintained under the thermal stresses of operation.

\section{Neutralisation of Ion Beam}

This ion beam is converted into a neutral beam by passing through the neutraliser gas cell which is usually fed with hydrogen. The protons are neutralised by electron capture, but unfortunately, the fast atoms so produced can be stripped by a further collision. cient.

\section{Beam Transmission} reaches the plasma.

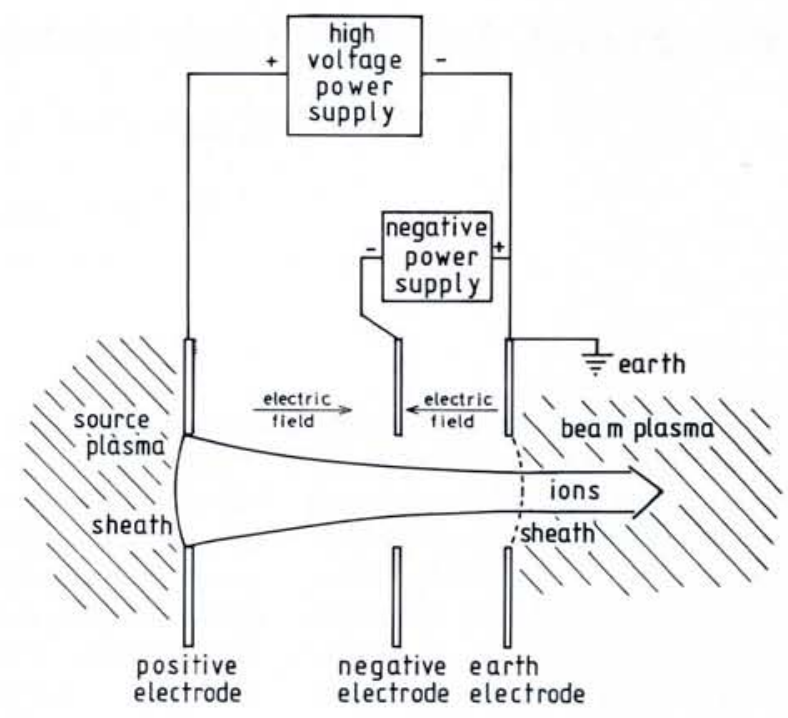

CAPTURE $\mathrm{H}_{\text {fast }}^{+}+\mathrm{H}_{2}=\mathrm{H}_{\text {fast }}+\mathrm{H}_{2}^{+}$ STRIPPING $\mathrm{H}_{\text {fast }}^{\circ}+\mathrm{H}_{2}=\mathrm{H}_{\text {fast }}^{+}+\mathrm{H}_{2}+\mathrm{e}$

These reactions cause negligible angular scattering of the ions and neutrals. In addition many fast molecular ions are dissociated and neutralised as above. With a thick gas target these reactions reach equilibrium with some fraction of neutrals in the beam depending on the particle energy. At low energy this fraction is high, but it falls with increasing energy. We shall see in the next section that small fusion experiments with low plasma density require low energy beams which are efficiently neutralised, but that large devices such as JET with high plasma density require particle energies of $>70 \mathrm{keV}$ and at these energies neutralisation is ineffi-

After neutralisation the unwanted ions are dumped usually by magnetic deflection. The beam duct is vigorously pumped to prevent further stripping, because any residual ions in the beam would be dumped by the magnetic fields of the fusion device in an uncontrolled manner. This can evolve sufficient gas effectively to block the neutral beam by the stripping reaction.

The neutral particles pass on unscattered into the fusion device where the entrace apertures are usually severely limited. Beam scrapers, consisting of refractory materials, scrape off that part of the beam which would otherwise intercept the duct and melt it. Nevertheless usually $>70 \%$ of the neutral beam

\section{Efficiency of Beam Production}

The overall efficiency of beam production is limited only by neutralisation and beam transmission. For low energy beams the overall power efficiency is typically $>40 \%$, but for high energy beams the efficiency can be as low as $10 \%$. This makes the power supply requirements formidable, and creates severe problems with waste power in the form of energetic particles, particularly for beam pulses lasting many seconds.

It is possible to recover the waste power in ions by means of a collector electrode connected to the high voltage electrodes. Another possibility is to accelerate $\mathrm{H}$ - ions since these can be efficiently neutralised even at high energy. The principles of both have been demonstrated, but have not so far been applied in a beam heating system.

\section{Interaction of the Neutral Beam}

As already mentioned we can divide the interaction of the beam with the plasma into three processes, ionisation of the neutrals, confinement of the energetic ions so produced and the subsequent surrender of the fast ion energy to the plasma. We shall look at these three processes in turn.

\section{Ionisation}

On entering the plasma the neutral beam is ionised by a number of atomic reactions, of which we list the most important:-

ELECTRON TRANSFER (charge exchange)

$$
\mathrm{H}_{\text {fast }}^{\circ}+\mathrm{H}_{\text {plasma }}^{+}=\mathrm{H}_{\text {fast }}^{+}+\mathrm{H}_{\text {thermal }}^{\circ}
$$

IONISATION BY ELECTRON IMPACT

$$
\mathrm{H}_{\text {fast }}^{0}+\mathrm{e}_{\text {plasma }}=\mathrm{H}_{\text {fast }}^{+}+\mathrm{e}_{\text {plasma }}+\mathrm{e}_{\text {cold }}
$$
IONISATION BY PROTON IMPACT

$$
\mathrm{H}_{\text {fast }}^{\circ}+\mathrm{H}_{\text {plasma }}^{+}=\mathrm{H}_{\text {fast }}^{+}+\mathrm{H}_{\text {plasma }}^{+}+\mathrm{e}_{\text {cold }}
$$

Here the subscripts indicate the source of the impacting projectiles, and the nature of the products. The energy exchanged in these relations is small, only a few eV, so the subscript 'fast' is used to describe the parent atom and the energetic ion produced by the collision. The subscripts 'plasma' and 'thermal' 


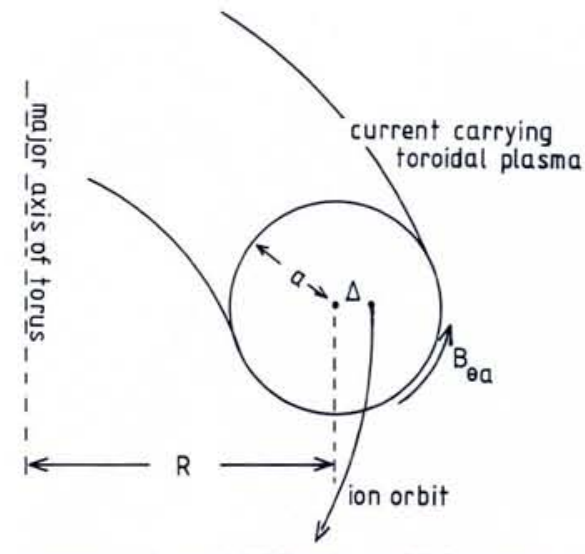

Fig. 4-A toroidal fast ion orbit in a current carrying toroidal plasma.

indicate particles with energies characteristic of the plasma temperature, whereas 'cold' indicates particles with energies of a few eV.

For neutrals with low energy $<30$ $\mathrm{keV}$, electron transfer dominates, and with high energy, $>50 \mathrm{keV}$, proton impact ionisation dominates. The total cross-section for conversion into fast ions falls steeply with increasing beam energy. This property is used to tailor the attenuation of the incoming beam to match the plasma density and dimensions so as to deposit the power in the desired region of the plasma. For plasmas such as in the Wendelstein stellara- tor ${ }^{2}$ ), where the plasma diameter is 20 $\mathrm{cm}$, beam energies $<20 \mathrm{keV}$ are required to deposit the neutral beam power in the centre of the plasma, but in JET an energy of $80 \mathrm{keV}$ for hydrogen beams is required.

\section{Confinement of the Energetic lons}

Having ionised the neutral beam, it is necessary to confine the energetic ions for times of $10 \mathrm{~ms}$ - 1s whilst they surrender their energy to the plasma. The equation of motion for a particle of charge $e$ and mass $m$ in electric fields $E$ and $B$ is: -

$$
m d V / d t=e(\mathrm{E}+\mathrm{V} \Lambda \mathrm{B})
$$

the vector cross product indicates that the vector of magnitude $B$ is multiplied by the component of $V$ perpendicular to $B$ with direction perpendicular to $B$ and $V$. If the ion Larmor radius is small we can decompose the ion motion into rapid Larmor gyration around a guiding centre and a slow drift of the guiding centre itself. Equations of motion of this guiding centre can then be solved numerically.

In a few cases it is possible to obtain simple illustrative results. One such case is illustrated in Fig. 4, where a toroidal plasma carries a current in the toroidal direction. There exists a toroidal orbit of

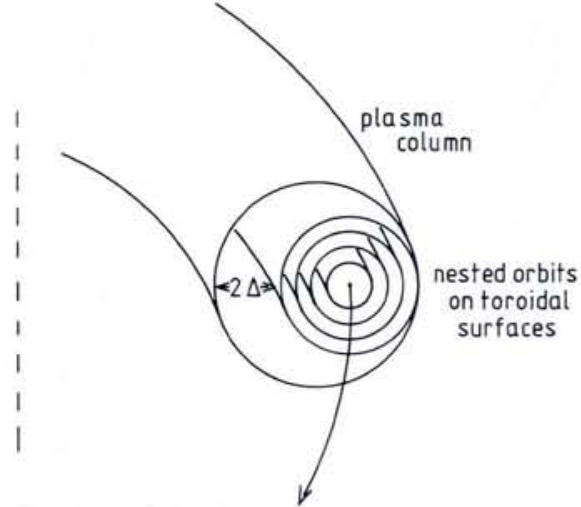

Fig. 5 - Other fast ion orbits in the geometry of Fig. 4 , which trace out nested toroidal surfaces.

radius $R+\triangle$ about the major axis where the centripetal force for toroidal velocity $V$ is balanced by the force on the particle due to the poloidal magnetic field $B_{\odot}$ generated by the plasma current.

i.e. $m V^{2} /(R+\Delta)=e V B_{\Theta}$ where $B_{\odot}=B_{\odot a} \Delta / a$.

Here $B_{\Theta}$ and $B_{\odot a}$ refer to the poloidal fields at the orbit and the edge of the plasma respectively; a uniform current density in the plasma is assumed. Thus when $\Delta<<R, \Delta=\rho_{\Theta}$ a/R, where $\rho_{\Theta}$ is the Larmor radius in the poloidal field at the edge of the plasma. Clearly if $\Delta>a$ the injected particle is not confined within the plasma column and will strike

H.G. Schuster

\section{Deterministic Chaos}

\section{An Introduction}

1984. XXII, 220 pages with 128 figures, 13 colored plates and 12 tables. Hardcover. DM 98.-/\$ 43.75. ISBN 3-87664-101-2

Daily experience shows that for many physical, chemical and biological systems small changes in the initial conditions lead to small changes in the outcome. But there are other cases: For a coin standing on end, a slight touch will suffice to make it fall and to determine the side on which it will fall. In recent years it has become clear that chaotic behavior resulting from a sensitive dependence on the initial conditions is not exceptional but typical of many systems. Examples are heart cells stimulated by a pacemaker, chemical reactions, particle accelerators or the onset of turbulence in fluids.
This book provides an introduction to this field from a physicist's point of view. It an-

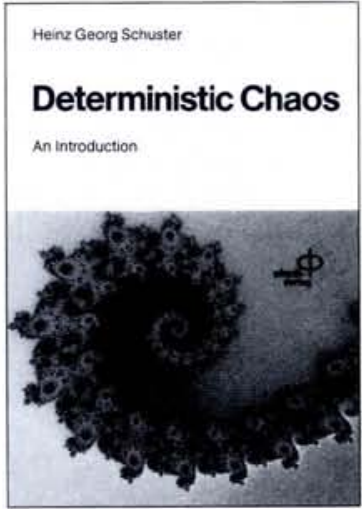
swers basic questions such as what is deterministic chaos? how is it generated? where does it occur? what can one learn from a chaotic signal? And it explains advanced concepts such as bifurcations, intermittency, Liapunov exponents, functional renormalization group and strange attractors. Moreover, the book contains 13 colored plates which show the surprising regularity and beauty of simulated chaotic events. 
some material surface of the device such as wall or limiter. Typical values for $\triangle$ lie in the range 5 to $10 \mathrm{~cm}$ for tokamak devices so fast ions can be confined. If we calculate the orbits of other ions injected in the toroidal direction we find they trace out toroidal surfaces nested about the orbit just discussed as shown in Fig. 5. There is a region of width $2 \triangle$ where fast ions are not confined, but by choosing the injection energy we can ensure that most neutrals are ionised in the confined region.

\section{Slowing Down of Fast lons}

The confined fast ions in the plasma slow down by Coulomb collisions with plasma electrons and ions and thereby surrender their energy. Close Coulomb collisions result in large angular scattering of the fast ion and large energy exchange. Distant Coulomb collisions are screened out by the intervening cloud of charged plasma particles. The collisions of interest here are intermediate between these extremes, with many weak encounters occurring all the time. The fast ions are slowed down gradually and scattered in angle gradually. We can describe this by the following equation for the change in fast ion energy $W$ :

$d W / d t=-(2 W / \tau)\left[1+\left(W_{c} / W\right)^{1.5}\right]$

$\tau$ is the time to slow down by collisions with electrons, and $W_{c}$ is known as the critical energy. Thus the first term describes friction from plasma electrons, and the second friction from plasma ions. At $W=W_{c}$ electron and ion friction contribute equally. $\tau$ and $W_{c}$ are readily calculated from collision theory, and typically lie in the range $1-100 \mathrm{~ms}$ and $10-80 \mathrm{keV}$. An injected ion therefore has to be confined for $100-1000$ orbit transits, and during this time gives comparable amounts of energy to plasma electrons and ions.

A few fast ions capture electrons from the background neutrals in the plasma, and can escape as fast neutrals. This is not a significant loss in energy terms, but provides a useful way of monitoring the slowing down by measuring the spectrum of escaping neutrals. Such spectra show that the slowing down is as described above.

\section{Calculation of the Effects of Beam Heating}

We have treated the interaction of the beam with the plasma as three independent pieces of physics. In fact, of course, the confinement of a fast ion depends on where it was ionised and on the collisional modification of the orbit during the slowing down. Calculations are often performed where a large number of particles are followed from the ion

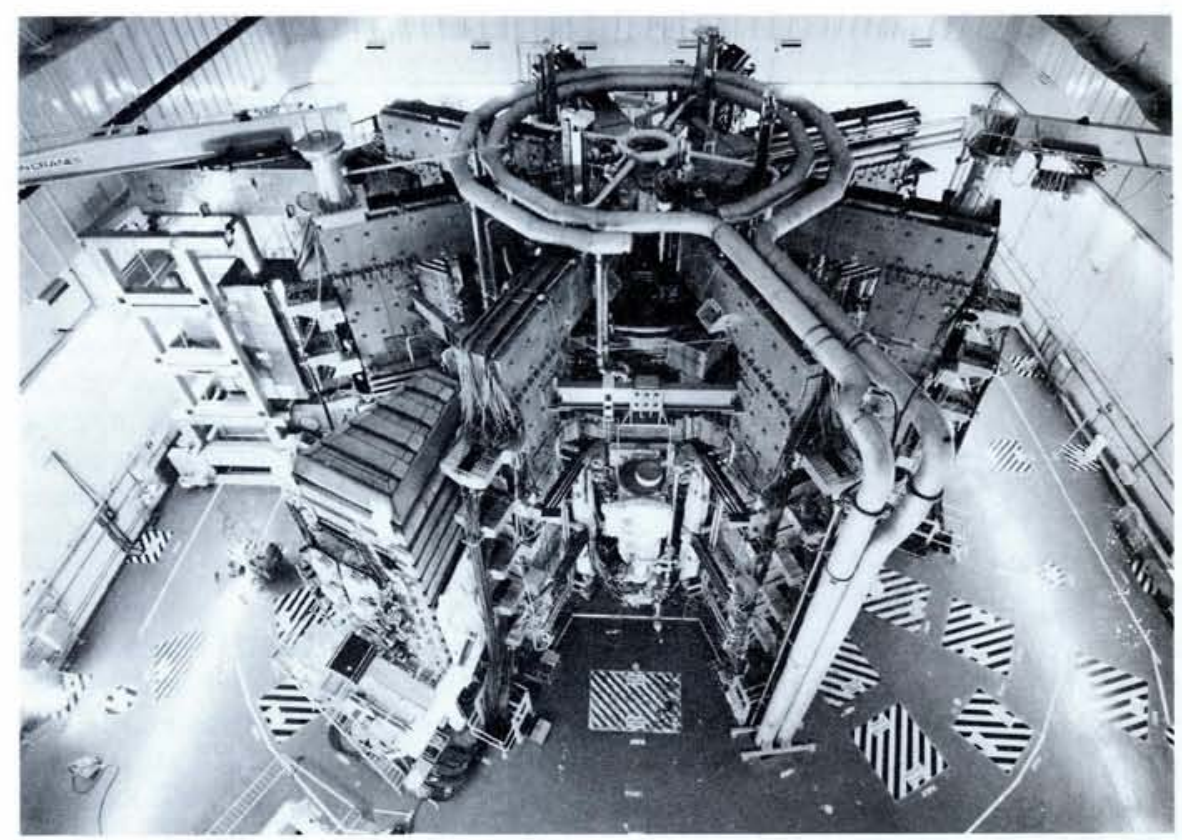

Fig. 6 - Overhead view of JET showing, left foreground, the initial stage of installation of the neutral injection system. One out of the eight sources is already in place.

(Photo JET)

source until they have finally slowed down in the plasma, and these demons trate that it is a very good approximation to treat separately ionisation, confinement and slowing down. Thus it is straightforward to calculate, for any case, the particle input, the power input to plasma electrons and to plasma ions, the spatial distribution of these inputs, and the expected spectrum of escaping neutrals. This last item provides a direct comparison between experiment and theory, and thereby confirms, indirectly, the estimates of power input.

A new equilibrium is reached when the losses from the plasma increase to balance the inputs from the neutral beam, and this generally occurs at a higher plasma temperature or energy content. However, if the heating mechanism itself triggers off new loss mechanisms from the plasma, the plasma temperature may even decrease. This can happen if, for example, the plasma is contaminated with impurities which radiate most of the power input, and for this reason the plasma must be 'clean'. Usually energy transport mechanisms, such as thermal conduction via the electrons, are responsible for the losses from the plasma, but the physics of these processes is only poorly understood, and it is difficult to predict precisely the plasma response to the power input from the neutral beams. Generally, the power inputs are calculated, the plasma response is measured, and empirical transport coefficients are then inferred. However, use of such data for making predictions, of the performance of JET for example, is fraught with danger.

\section{Summary}

The physics of neutral beam injection is straightforward involving only classical physics, both in beam production and in interaction with the plasma. The plasma physics is crucial only in the plasma response to the power input, and this is a productive line of current research. The technology has so far kept pace with the demands of experiments, providing more powerful beams at higher particle energies. Unfortunately at these higher energies neutral beams can only be generated at modest efficiencies.

At present other heating schemes are being tested on a large scale (for example radiofrequency heating). These schemes offer higher efficiencies, which is attractive for large costly experiments, but involve much plasma physics in the interaction with the plasma, which is not so attractive for plasma confinement physics experiments. The technology of high efficiency neutral beam generation at high energies is being developed, albeit on a modest scale, and could be incorporated in the next large fusion experiment if required. Looking even further ahead, neutral injection is one possible way of igniting a fusion reactor, but there are many problems to solve before then!

\section{REFERENCES}

1. Gibson A., 'The Scientific Programme of JET' Europhysics News 14 (1983) 4.

2. Consoli T., 'RF Supplementary Heating for Toroidal Reactors', Europhysics News 9 (1978) $1 / 2$.

3. Wobig H., 'Stellarators', Europhysics News 13 (1982) 8/9. 